\title{
Effect of using different types of methods for the derivation of elastic modulus of bone - A critical survey
}

\author{
Zartasha Mustansar $^{1,2}$, Arslan Shaukat ${ }^{1}$ and Lee Margetts ${ }^{2,3,4}$ \\ ${ }^{1}$ National University of Sciences and Technology (NUST), H-12, Islamabad, Pakistan. \\ ${ }^{2}$ School of Earth, Atmospheric and Environmental Science, The University of Manchester, Williamson Building, Oxford Road, Manchester, \\ M13 9PL, UK. \\ ${ }^{3}$ Research Computing, The University of Manchester, Devonshire House, Oxford Road, Manchester, M13 9PL, UK. \\ ${ }^{4}$ Oxford eResearch Centre, University of Oxford, 7 Keble Road, Oxford, UK.
}

\begin{abstract}
The purpose of this paper is to critically review and address the cause of variation in the values of elastic modulus (E) of bone. Properties used for materials (especially) bones is one of the crucial factors in modeling and simulation experiments with sensitive procedures such as Finite Elements. The values of elastic modulus acquired from secondary databases, literature and online repositories differ in many ways. This is due to an underlying fact that different methods are used for one definite set of species and vice versa. According to a human understanding, this might bar the perceptive of use of elastic modulus in the research when (especially) insightful procedures are in question for example Finite Element Analysis (FEA) of materials with sensitive procedures. The means and the methods, through which the values are obtained, do not follow a standard methodology. Thus, this area needs attention. Despite the advances in technology and state-of-the-art methods in the field of biomechanics and biomedical engineering there is a need to formulate a standard method which can produce_reliable set of E-values. Through this paper, we seek to raise an important scientific question as to what systemic methodology should be adapted to achieve a value of elastic modulus that is not only reliable, but reasonable as well. The paper also, focus briefly on the sources of error responsible for this variation and the significance of elastic modulus values in important numerical methods such as Finite Elements (FE).
\end{abstract}

\section{Introduction and Background}

Young's modulus or elastic modulus is one of the fundamental inputs in the constitutive analysis of bones using Finite Element Method (FEM). It is therefore important (at minimum) to get the linear part of the FE analysis correct. Elastic modulus can be obtained experimentally from a material that is subjected to a load, and the strain and stress are determined concurrently [1].

Bone, consist of combination of ingredients, details of which can be obtained from [2]. The shape of bone is determined by the arrangement of the HA crystals. The arrangement of the collagen fibrils and HA crystals enhances bone strength [3]. Due to a higher elastic modulus of HA crystals, bone is stiffer to bear the external fractures [4]. Bone has two distinct components. Cortical bone which constitutes the outer thick layer providing strength to the inner porous structure. Whereas the shape of trabeculae bone is related to porosity. Trabecular orientation is commonly studied nowadays mainly as a composite with anisotropic and open porous cellular solid from an engineering point of view [5-7].
Bones possesses specific material properties. They, fundamentally help determining an overall mechanical behaviour. The Young's modulus and the Poisson ratio are widely considered. The Young's modulus is already described above and the Poisson ratio $(\nu)$ is the negative ratio of transverse strain to the longitudinal strain [1]. When a specimen is loaded in compression, it will shorten in the direction of the load and will elongate in width. In other case if the specimen is loaded in tension it enlarges in the direction of the load but become narrower in width [8]. The ratio of change of these quantities describes how Poisson ratio is related to bones and usually ranges from $0.1-0.33$. A vast variation can be seen in the results of the values of Young's modulus (from $1 \mathrm{GPa}-20 \mathrm{GPa}$ ) in different analytical bone models (Please see section methods). One of our previous papers [9] discusses the effect of different loading tests in the derivation of modulus of elasticity.

This paper particular address one of the factors i.e.types of loading tests used for the derivation of elastic modulus (E) value. Another objective of this paper is to report an evaluation of the degree of refinement of 
biomechanical experimental data. Despite the advances in technology and state-of-the-art methods in the field of computational mechanics and biomedical engineering in general, there is a need to devise a standard method which can validate predicted results from the numerical methods.

\section{Methodology}

This section describes the rationale behind the survey in order to identify published literature for the determination of bone's Young's modulus. Authors points out how the selection of different testing methods for the derivation of material properties of bone may result in huge variation. We use a search method with a typical inclusion and exclusion criteria. Search methods consist of the relevant articles retrieved from published literature. Any information with incomplete citation on the web is excluded. The discussion includes only peer- reviewed journals, conference papers, conference proceedings, books and edited book sections, high impact letters and internationally accepted reports to address various data sets that report a typical value for elastic modulus. In this article, we will only focus on one factor i.e. relationship of testing method on elasticity (Elasticity-Type of method).

\section{Results and Observations}

Authors investigated, methods reported in literature to find out what should be the reasonable way of choosing one method over another while handling procedures used for the derivation of Young's modulus in bones. This section is divided in two parts, first the methods in literature used for derivation of elastic modulus are reported, then the authors discuss their own recommendations briefly.

\subsection{Methods used in Literature}

In this subsection, author describes various methods used in the literature for derivation of elastic modulus. For each method, a review of how the method works (principle of method); some limitations where required and for what purpose was it used, is described. In the end, we discuss the values of Young's modulus typically obtained from these methods (Figure 1) and then a profound discussion on why the variation exists in the literature library.

\subsubsection{Compression}

A compression test determines behaviour of materials under crushing loads. The specimen is compressed and deformation at various loads is recorded. It is expressed in Eq. (1) as:

$$
\sigma_{\text {comp }}=\frac{F}{A_{\text {cort }}}
$$

where $\sigma_{\text {comp }}$ is compressive stress, $F$ is the applied force and $A_{\text {cort }}$ the cross-sectional cortical area [10]. Compressive stresses and strains are calculated and plotted as stress-strain diagram which is used to determine elastic limit ;yield point, yield strength and for some materials compressive strength.

Compression has been used in studies involving bones, by [8, 11-14] and [15]; biomedical research Boyd et al., [16]; [6, 17-19] in natural sciences [20]. In some studies it is used for the study of local deformation [16],[21].

\subsubsection{Tension}

A tensile test, also known as tension test, is probably the most fundamental type of mechanical testing in materials. The test is performed by pulling the material to determine how the material will react to forces which are applied in tension test. Pulling material will give the strength of the material as well as strain (i.e. how much the material went elongation). The tension test usually is plotted as a graph showing the relationship of stress (applied force) to the strain (change in length/observed elongation) - the gradient the curve determines the value of the Young's modulus. It is a widely used method for testing bones [19, 22-24].

\subsubsection{Bending}

This test is basically used to test the ductility, strength, stiffness and modulus of elasticity. Based on the type of testing (3-point and 2-point) the direction of forces can be located in order to predict stress-strain response of the material. The gradient of the stress-strain curve obtained, gives the value of the Young's modulus of the material. Mostly the specimens with long cylindrical shapes are tested in bending because, it is simple to set up and predict reasonably accurate response.

\subsubsection{Buckling}

Buckling is a mathematical instability which leads to a failure mode in a specific material. This phenomenon usually occurs in thin bones and they tend to break the structure into two. It is a kind of failure mode that causes incapability to the function of the bone. Theoretically, it is caused by a bifurcation (in two). Detailed example can be seen in [25].

\subsubsection{CT scanning}

Computed tomography is a non-destructive evaluation technique that allows the internal structure of an object to be imaged by reconstructing the spatial distribution of the local linear X-ray absorption coefficients of the materials/phases contained within [26]. In one of the studies [27] a method of theoretical derivation was proposed. The method aimed to determine a relationship between voxel-specific tissue density and micro CT scan data (i.e., microCT derived tissue mineral density (TMD), $\mathrm{mgHA} / \mathrm{cm} 3$ ) and bone constituent properties. 'Voxel 
based tissue density derivation' is used from the scan data to derive an equivalent hydroxyapatite density using a manufacturer-specific calibration curve. This equivalent mineral density is then employed to determine elastic modulus of the individual mineral content. With an increase in the tissue density Young's modulus increases, which was one way of deriving value for the modulus of elasticity. For details of the method reader is referred to [27].

\subsubsection{Ultrasonic}

Natural resonance frequencies can be employed for a single small sample of bone to measure the elastic modulus of material. Such method can be seen in [28]. Typically this method is based on the measurement of wave propagation time in specimen [29] and then deriving Young's modulus. For detail on methodology reader is directed to [37].

This method outputs a 'dynamic Young's modulus $E$ [MPa]' which is calculated by using Eq. (2):

$$
E=v^{2} \rho \text {. }
$$

\subsubsection{Multi-scale modelling}

The multi-scale modelling approach consists of successive homogenization steps. Homogenization is the method of treating different scales/levels within the same specimen at individual phases. The method is described in [30]. In this study the trabecular bone was modelled as a nano-composite with hierarchical structure. This model was then used to predict its elastic properties at different structural scales. Their analysis made used of bottom-up multi-scale approach such that the starting phase is initiated with nanoscale (mineralized collagen fibril) and therefore onwards moving up to the sub-microscale (single lamella), microscale (single trabeculae) and mesoscale (trabecular bone) levels. Other examples can be seen in $[4,30-32]$.

\subsubsection{Finite Element Method}

By using CT based data, it is possible to convert a digital model into a numerical model. For example using Simpleware suite Ltd,. The numerical model (FE- based model) is used to determine the Young's modulus by testing the specimen under compression, tension and/or bending by using a computer solver (e.g. Abaqus). The constitutive models therefore obtained from this method are used to calculate elastic modulus via stress-strain curves.

\subsubsection{Cantilever bending with finite element analysis}

This method uses cantilever beam type bone specimen. The actual material properties are obtained by using the initial input estimates of the bone tissue material properties which were later employed in the finite element model. Difference between the calculated finite element displacements and the experimentally observed displacements help in the determination of actual Young's modulus.

\subsubsection{Back-calculating from finite element models}

By using the deformation values i.e. the change in length to the original length of the samples, finite element models can be used to determine the elastic properties 'Young's modulus' of the materials. Mente and Lewis [33] used cantilever bending with finite element analysis for elastic properties determination and Williams and Lewis [34] used back-calculation from FE models.

\subsubsection{Micro hardness}

This method uses the static indentations made with loads not exceeding than $1 \mathrm{~kg}$. In bone mechanics this method is seen in [35]. However, it requires a metallographic finish for the surface to be tested. Smaller loads tend to use higher finish.

\subsubsection{Using stochastic operators}

Wille et al., [36] used the stochastic operations to pool data cited in the literature based on the relationship between Young's modulus and bone density. All the experiments were pooled over time regarding the E-d (Young's modulus-density) relationship a stochastic relationship between them was developed.

\subsubsection{Nanoindentation}

Nanoindentation is used to measure the intrinsic elastic properties of individual micro structural bone components (here trabecular bone and cortical bone) in various directions. This method uses a load which is applied to an indenter of known properties. This leads to a formation of residual impression in the test material. The dimensions of the residual impression and the contact area are then measured directly to determine the hardness of the specimen.This method has the ability to give a spatial resolution of around $1 \mu \mathrm{m}$ [37] to determine bone's mechanical properties and to achieve bone's nearly accurate young's modulus in several directions because of the highly anisotropic [6, 38-44] nature of trabecular bone.Nanoindentation also has the capability of producing large indent matrices in a very short period of time with stability especially for mapping the mechanical properties of complex surfaces [45]. Moreover it uses advance depth sensing indentation method, to produce a load displacement curve which is used to measure the stiffness and elastic properties of the sample using various approaches [46].

\subsubsection{Digital volume correlation (DVC)}

Digital volume correlation (DVC) is a measurement technique that quantifies strains throughout the interior of a specimen unlike traditional strain gauge methods where 
only few points on the surface of the materials are considered. Through DVC it is possible to generate stressstrain curve, using a software suite like Daviz. The gradient of stress-strain curve gives value of the Young's modulus.

\subsection{Recommendations}

In comparison to all the methods listed above, authors reserves their own opinion on how a large sets of different methods could affect the values of the elastic modulus obtained.

Through careful investigation, at first all the values of elastic modulus of bones are fetch from the literature against different types of species and methods used are populated in the form of the graph/illustration as shown in Figure 1. Figure 1 reviews the author's own survey on trend/behavior of huge variation in the values of Young's modulus from the literature of bones. It is clear from the chart that the variation in the method of determination yields a large variation in the spread of elastic modulus values. Eventually, all the biomechanical analyses which require elastic modulus as one of their important parameters can be affected. This means, a careful consideration is required in choosing a specific value for the elastic modulus in any type of analysis where elastic modulus is one of the inputs. To be more precise, a systematic procedure is inherent to introduce so that this variation can be doomed to a typical consistency level as required originally.

The bone's elasticity is important because, it gives the bone ability to dissipate energy during deformation phenomena's. Methods like Finite Elements, which requires the value of elastic modulus as one of the major inputs predicts results of simulation on the basis of Evalue that is used as an input. If one value goes wrong the whole simulation will result into an inaccurate and ambiguous situation. Apparently, the criticality of this basic factor is under estimated and it might result into the situations which do not relate to any real physical phenomena's at all.

\subsection{Limitations}

In this study, detailed studies on review of the papers that uses Finite Element Method as a method of derivation is not reported.

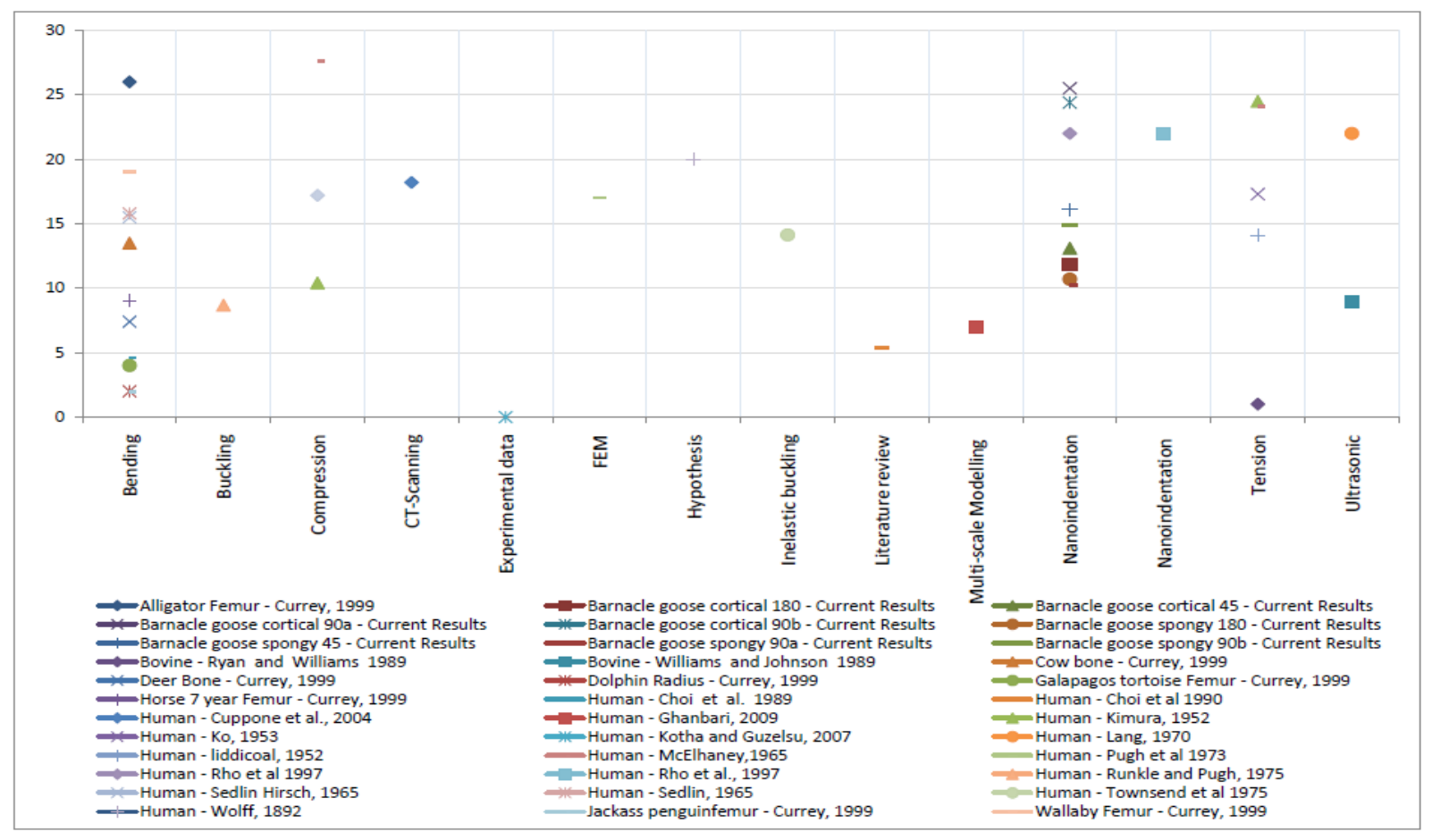

Figure 1. Chart showing spread of material properties compared to results obtained from Barnacle goose (Branta leucopsis) specimen.

This might have helped to validate our argument on how the variation in the values of elastic modulus, might affect the simulation results. This is something we are hoping to include in our next coming papers.

Secondly, the study takes into account only the affect of loading methods, used for the derivation of elastic modulus. Such type of tests were briefly discussed in one of our previous papers as well [9] but only for the non homogenous materials and the methods were also not discussed in much detail. Here we report almost every single method found from literature to address variation in the elastic modulus.

Additionally, apart from the types of loading tests, there are several other factors, for example, type of mechanical environment, bone density, bone age, specie type, physical conditions in which bones are prepared for 
the mechanical testing and treated bones, which adds to the variation among the E-values. It might be good to include those studies as well in future.

\section{Discussions and Conclusions}

Due to the complex geometry of bone it is not possible to measure the exact Young's modulus. The way bone samples are prepared and the methods used for testing, equally contribute to the variation of the elastic modulus values achieved for bones. A correlation between methods used to determine Young's modulus with respect to the factors affecting $E$ value is a 'must' required procedure.

Different testing methods tend to yield different values of Young's modulus. For example, a bone in compression will yield a different value of Young's modulus compared to the bone tested in tension under the same physical conditions. Therefore, it is important to hypothesize a 'generalized method.

By using similar methods and techniques to find an answer for material property can be helpful in maintaining a consistency and reliability among these values. Authors also conclude that there is a need to hypothesize a 'generalized method' which can give reasonable results under similar physical conditions (for example, specific laboratory standard should be practiced at least for one type of bone specimen.

As said in limitations, there are number of factors which can affect the method of determining value of elastic modulus that may in turn affect the original elastic properties of bone when it was alive. For example a healthy will function much better than a damaged bone whereas a damaged bone is likely to cause a functional disability. So if the condition of specimen changes, its functionality also changes.

The factors effecting value of $\mathrm{E}$ can be biological, mechanical or experimental. These parameters, if considered in detail in future will reduce the risk of false generation of data for the elastic modulus of bone. Increasing water loss from bones also make them lose elasticity due to breaking bonds within the elastic trabeculae. It should also be noted that bigger animals tend to have stronger bones due to thicker trabeculae [47]. This means size of the bone might also be one limiting factor as well to generate a real values for Young's modulus.

In this paper we have presented the scheme of the method to be used in future for the selection of Young's modulus in numerical methods (specially). This is quite a challenging task though crucially very basic. Therefore there is a need to validate this by using finite element method which is a reliable method to address such problems virtually by replicating the reality in a virtual environment.

Therefore we propose a new methodology, by using Evalues from ten different species initially. All specie bones will be prepared under different physical conditions and mechanical testing methods. Additionally, all bones will be of different age and bone density. Under such conditions, a finite element analysis will be conducted under one set of boundary conditions and i.e. force and the base conditions remains the same throughout the ten species. The analysis will entail the difference in the deformation scale factor and the amount of stress due to different values of elastic modulus (E). To our opinion, this will be a very useful step to address any kind of ambiguity in the future of biomechanics.

\section{Acknowledgments}

Authors would like to thank The Microsoft-Dorothy Hodgkin Postgraduate award (FA01546) for their financial support.

\section{References}

1. A. Sharir, et al., "Whole bone mechanics and mechanical testing," The Veterinary Journal, vol. 177, pp. 8-17, (2008).

2. J. D. Currey, Bones: Structure and Mechanics.: Oxford, Princeton University Press,(2000).

3. J. D. Currey, "Collagen and the Mechanical Properties of Bone and Calcified Cartilage," in Collagen structure and mechanics, ed: Springer US, (2000), pp. 397-420.

4. J. Ghanbari and R. Naghdabadi, "Nonlinear hierarchical multiscale modeling of cortical bone considering its nanoscale microstructure," Journal of Biomechanics, vol. 42, pp. 1560-1565, (2009).

5. P. J. Kolston, "Finite element modeling: a new tool for the biologist.," Philosophical Transactions of the Royal Society (Series A: Mathematical, Physical and Engineering Sciences), vol. 358, pp. 611-631, (2000).

6. T. M. Keaveny, et al., "Biomechanics of trabecular bone," Annu. Rev. Biomed. Eng., vol. 3, pp. 307-33, (2001).

7. S. Sobieszczyk, et al., "FEM Approach to estimate the behaviour of Bio-Composite metal-surface coating systems.," Advances in Materials Sciences, vol. 8, pp. 166-172, (2008).

8. A. Benoit, et al., "3D analysis from micro-MRI during in situ compression on cancellousbone," Journal of Biomechanics, vol. 42, pp. 2381-2386, (2009).

9. R. Khan and Z. Mustansar, "Reliability of using elastic modulus for non- homogeneous materials.," in 6th International Conference on Bioscience, Biochemistry and Bioinformatics (in press), Pattaya, Thailand, (2016).

10. Gere J and G. B., "Mechanics of materials.," Stamford, CT: Cengage Learning.,(2012).

11. R. Müller, et al., "Micro-compression: a novel technique for the nondestructive assessment of local bone failure," Technology and Health Care, vol. 6, pp. 433-444, (1998).

12. S. Nagarajaa, et al., "Trabecular bone microdamage and microstructural stresses under uniaxial compression," Journal of biomechanics, vol. 38, pp. 707-716, (2005).

13. P. J. Thunrner., et al., "High-Speed Photography of Human Trabecular Bone during Compression," in In: Material Research Societyvol. 874, ed, (2005).

14. R. Das and W. Cleary, Paul. , "Uniaxial Compression Test And Stress Wave Propagation Modelling Using Sph," CSIRO Mathematical and Information Sciences, Clayton, Victoria 3168, Australia15-16 December 2006 (2006).

15. R. Rodríguez-Martín, et al., "Use of electronic speckle pattern interferometry in the detection of fatigue failure in high strength steels," Engineering Failure Analysis, vol. 17, pp. 226-235, (2010)

16. S. Boyd, et al., "Measurement of cancellous bone strain during mechanical tests using a new extensometer device," Medical Engineering \& Physics, vol. 23, pp. 411-416, (2001).

17. E. Perilli, et al., "Structural parameters and mechanical strength of cancellous bone in the femoral head in osteoarthritis do not depend on age," Official journal of the international bone and mineral society, vol. 41, pp. 760-768, (2007) 
18. G. Y. H. Lee and C. T. Lim, "Biomechanics approaches to studying human diseases," Trends in Biotechnology,vol. 25, pp. 111-118, (2007).

19. S. P. Kotha and N. Guzelsu, "Tensile behavior of cortical bone: dependence of organic matrix material properties on bone mineral content.," Journal of Biomechanics, vol. 40, pp. 36-45, (2007).

20. D. D. Deligianni and C. A. Apostolopoulos, "Multilevel finite element modeling for the prediction of local cellular deformation in bone.," Biomechanics and Modeling in Mechanobiology, vol. 7, pp. 151-159, (2008)

21. E. Verhulp, et al., "Indirect determination of trabecular bone effective tissue properties using micro-finite element simulations "Journal of biomechanics, vol. 41, pp. 1479-1485, (2008).

22. D. R. Carter, et al., "Tensile fracture of cancellous bone," Acta Orthop. Scand, vol. 51, pp. 733-741, (1980).

23. S. J. Kaplan, et al., "Tensile strength of bovine trabecular bone," Journal of biomechanics., vol.18, pp. 723-727, (1985).

24. C. B. Ryan and J. L. Williams, "Tensile testing of rodlike trabeculae excised from bovine femoral bone. ," Journal of Biomechanics, vol. 22, pp. 351-355, (1989).

25. X.-P. Zheng, et al., "Determining the elastic modulus of thin films using a buckling-based method: computational study," Journal of physics D: Appplied physics., vol. 42, (2009).

26. J. C. Elliott and S. D. Dover, "X-ray Microtomography," Journal of Microscopy-Oxford, vol. 126, (1982).

27. W. D. Wagner, et al., "Deriving tissue density and elastic modulus from microCT bone scans," BONE, vol. 49, pp. 931-938, (2011).

28. R. B. Ashman and J. Y. Rho, "Elastic modulus of trabecular bone material. ," Journal of Biomechanics, vol. 21, (1988).

29. H. Nìmcová, et al., "Comparison of methods for dynamic young's modulus determination in gypsum materials," presented at the The 4th International conference: Modelling Of Mechanical And Mechatronic Systems 2011, The 4th International conference, (2011).

30. E. Hamed, et al., "Multi-scale modelling of elastic moduli of trabecular bone," Journal of royal society interface, vol. (in press), (2012).

31. D. Porter, "Pragmatic multiscale modelling of bone as a natural hybrid nanocomposite," Materials Science and Engineering, vol. 365, pp. 38-45, (2004).

32. V. Sansalone, et al., "Multiscale modelling of mechanical properties of bone: study at the fibrillar scale," Comptes Rendus Mecanique vol. 335, pp. 436-442, (2007).

33. P. L. Mente and J. L. Lewis, "Experimental method for the measurement of the elastic modulus of trabecular bone tissue.," Journal of Orthopaedic Research, vol. 7, (1989).
34. J. L. Williams and J. L. Lewis, "Properties and an anisotropic model of cancellous bone from the proximal tibial epiphysis," Journal of Biomechanics and Engineering, vol. 104, pp. 50-56, (1982).

35. R. Hodgskinson, et al., "Hardness, an indicator of the mechanical competence of cancellous bone.," Journal of Orthopaedic Research, vol. 7, pp. 754-758, (1989).

36. H. Wille, et al., "Prediction of the mechanical response of the femur with uncertain elastic properties," Journal of Biomechanics, vol. 45, pp. 1140-1148,( 2012).

37. J. Y. Rho, et al., "Young's modulus and hardness of trabecular and cortical bone in various directions determined by nanoindentation.," in In: Transactions of the 43rd Annual Meetings of the Orthopaedic Research Society., (1997), p. 891.

38. Yang G, et al., "The anisotropic hooke's law for cancellous bone and wood," Journal of Elasticity, vol. 53, pp. 125-46, (1999).

39. J. Seto, et al., "Tough Lessons From Bone: Extreme Mechanical Anisotropy at the Mesoscale," Advance Function Materials, vol. 18, pp. 1905-1911, (2008).

40. S. Dendorfer, et al., "Anisotropy of the fatigue behaviour of cancellous bone, Journal of Biomechanics," Journal of Biomechanics, vol. 41, (2008).

41. P. Fratzl, "Collagen and the Mechanical Properties of Bone and Calcified Cartilage," in Collagen structure and mechanics, J. D. Currey, Ed., ed: Springer US, (2008), pp. 397-420.

42. J. Y. Rho, et al., "Mechanical properties and the hierarchical structure of bone," Medical Engineering and Physics, vol. 20, pp. 92-102, 1998

43. J. Zhang, et al., "Mechanical property determination of bone through nano- and micro-indentation testing and finite element simulation.," Journal of Biomechanics, vol. 41, pp. 267-275, (2008).

44. P. K. Zysset, et al., "Elastic modulus and hardness of cortical and trabecular bone lamellae measured by nanoindentation in the human femur," Journal of Biomechanics, vol. 32, pp. 1005-12, (1999).

45. X. N. Randall, et al., "Nanoindentation analysis as a 2-dimensional tool for mapping mechanical properties of complex surfaces. ," Journal of Materials Research, vol. 24, (2009).

46. C. W. Oliver and G. M. Pharr, "An improved technique for determining hardness and elastic modulus using load and displacement sensing indentation experiments," Journal of Materials Research, vol. 7, (1992).

47. M. Doube, et al., "Trabecular bone scales allometrically in mammals and birds," Proceedings of Royal society, (2011). 